\title{
The Effects of Partial Acetylation on the Mechanical Properties of Poly(vinyl alcohol)
}

\author{
Hiroshi Ochial, Hideko Fujir, ${ }^{*}$ Masami Watanabe, \\ and Hitoshi Yamamura \\ Department of Chemistry, Faculty of Science, Hiroshima University, \\ Hiroshima, Japan.
}

(Received February 14, 1974)

\begin{abstract}
The mechanical properties of partially acetylated poly(vinyl alcohol) were studied by dynamic measurements over a wide temperature range. After partial acetylation, the $\alpha_{\mathrm{c}}$-loss peak disappeared and the $\beta_{\mathrm{c}^{-}}, \alpha_{\mathrm{a}}-$, and $\beta_{\mathrm{a}}$-loss peaks shifted to lower temperatures and showed an increase in their peak heights. The $\beta_{c}$-loss peak of the highly acetylated samples merged into the $\alpha_{\mathrm{a}}$-loss peak. There was also a linear relation between the density and the temperature of the $\beta_{\mathrm{a}}$-loss peak for the partially acetylated samples. These results are discussed in terms of the disordering of the crystalline structure, breakdown of the hydrogen bonds, and increase in the local mobility of the chain segments by partial acetylation.

KEY WORDS Mechanical Relaxation / Partial Acetylation / Poly(vinyl alcohol) / Poly(vinyl acetate) / Density / Hydrogen Bond / Local Mobility / Activation Energy /
\end{abstract}

It is well known that poly(vinyl alcohol), PVA, which is normally prepared by the hydrolysis of poly(vinyl acetate), PVAc, obtained from the conventional radical polymerization, shows a high crystallinity even though it is essentially atactic. ${ }^{1-3}$ This high crystallinity of atactic PVA may be ascribed to the small difference between the van der Waals radii of the $\mathrm{H}$ atom and the $\mathrm{OH}$ group, and the strong hydrogen bonds between the $\mathrm{OH}$ groups on neighboring chains, which tend to pack the molecules in a regular structure in spite of the lack of stereoregularity. ${ }^{1}$ Thus, quite a large number of reports about this polymer have been published dealing with the mutual relations between the crystallinity, the mode of molecular motions, the hydrogen bonds, and so on. ${ }^{4-12}$ By extensive mechanical and dielectric studies, ${ }^{8}$ it was found that five loss peaks could be observed in PVA $\left(\alpha_{\mathrm{c}^{-}}, \alpha_{\mathrm{a}^{-}}, \beta_{\mathrm{c}^{-}}, \beta_{\mathrm{a}^{-}}\right.$, and $\beta_{\mathrm{w}}$-loss peaks). Of these loss peaks, the $\alpha_{\mathrm{c}}$-loss peak is generally ascribed to the translational or twisting mode of the chain segments along or around the chain axis in the crystalline phase, and the

* Present address: Department of Chemistry, University of the Sacred Heart, Shibuya-ku, Tokyo, Japan. $\beta_{\mathrm{c}}$-loss peak is ascribed to the motions of the chain segments in the imperfect crystalline phase. The $\alpha_{\mathrm{a}}$-loss peak evidently results from the micro-Brownian motions of the long chain segments in the amorphous phase. The $\beta_{\mathrm{a}}$-loss peak seems to arise from the local motions of the chain segments, including the inter- and intramolecular hydrogen bonded portions; the $\beta_{\mathrm{w}}$-loss peak arises probably from the absorbed water moisture. However, there is no clear knowledge of the mechanisms of the $\beta^{\prime}$-loss peaks.

Generally, when poly(vinyl alcohol) is partially acetylated, its crystalline structure is considerably disturbed and at the same time the melting point is depressed. These phenomena are ascribed to the decrease in the crystallinity resulting from the bulkiness of the acetyl groups introduced and also from the decrease in the number of hydrogen bonds, which perform the main role in the crystallization of PVA chains. ${ }^{13}$ From this point of view, we discuss the effects of partial acetylation on the relaxational processes of PVA from the dynamic viscoelastic measurements. 


\section{EXPERIMENTAL}

The sample poly(vinyl alcohol), PVA, used in the present study, was prepared by the complete saponification of conventional PVA; the degree of polymerization was about 2000. Partially acetylated PVA's were obtained by the conventional acetylation method from purified PVA with a suitable concentration of acetic acid or acetic anhydride in the presence of catalystic hydrochloric acid for $50 \mathrm{hr}$ at $60^{\circ} \mathrm{C}^{14}$ Then they were purified by dialysis and electrodialysis to remove ionic impurities. The degree

Table I. Some characteristics of sample polymers

\begin{tabular}{|c|c|c|c|c|c|}
\hline \multirow{2}{*}{ Samples } & \multirow{2}{*}{$\begin{array}{c}\text { Degree of } \\
\text { acetyla- } \\
\text { tion, } \\
\text { mol\% }\end{array}$} & \multicolumn{2}{|c|}{ Annealing } & \multirow{2}{*}{$\begin{array}{c}\text { Density, } \\
\mathrm{g} / \mathrm{cm}^{3}\end{array}$} & \multirow{2}{*}{$\begin{array}{c}\text { Crystal- } \\
\text { linity, } \\
\%\end{array}$} \\
\hline & & ${ }^{\circ} \mathrm{C}$ & $\mathrm{hr}$ & & \\
\hline PVA & 0 & 0 & 0 & 1.301 & 45.6 \\
\hline PVA & 0 & 120 & 3 & 1.310 & 58.4 \\
\hline PVAc-1 & 1.3 & 120 & 3 & 1.288 & 28.2 \\
\hline PVAc-12 & 12.0 & 120 & 3 & 1.272 & - \\
\hline PVAc-28 & 27.9 & 90 & 3 & 1.247 & - \\
\hline PVAc-38 & 37.9 & 90 & 3 & 1.235 & - \\
\hline PVAc-59 & 58.9 & 60 & 3 & 1.218 & - \\
\hline PVAc-69 & 69.3 & 60 & 3 & 1.121 & - \\
\hline PVAc-80 & 79.6 & 60 & 3 & 1.121 & - \\
\hline PVAc-100 & 100.0 & 60 & 3 & 1.194 & - \\
\hline
\end{tabular}

of acetylation was estimated by chemical analysis of the acetyl group. ${ }^{15}$ The sample polymer was cast into thin films about $50 \mu$ in thickness by slow evaporation of about 5-\% solution, with suitable solvents, on a mercury surface. The films thus obtained were dried for a week at room temperature under reduced pressure and then stored in a vacuum desiccator with phosphourus pentoxide. The heat treatments for the sample were carried out at $120^{\circ} \mathrm{C}\left(90^{\circ}\right.$ or $60^{\circ} \mathrm{C}$ for the highly acetylated samples) for $3 \mathrm{hr}$ in vacuo. The sample density was determined by the flotation method with $\mathrm{CCl}_{4}$-xylene and $\mathrm{CS}_{2}$-hexane mixtures of various concentrations at $25^{\circ} \mathrm{C}$. For the slightly acetylated samples, the degree of crystallization was estimated from the density value. These characteristics of the samples are listed in Table I along with the acetylation and annealing conditions.

The apparatus used here was a forced oscillation-type viscoelastic spectrometer (Iwamoto Seisaku-sho Co., Ltd.). The dynamic Young's modulus $E^{\prime}$, loss modulus $E^{\prime \prime}$, and $\tan \delta$ for each sample were obtained as a function of temperature over the range of $-150^{\circ}$ to $200^{\circ} \mathrm{C}$ at fixed frequencies of 5,30 , and $80 \mathrm{~Hz}$. Care was taken to ensure the absence of humidity during the measurements.

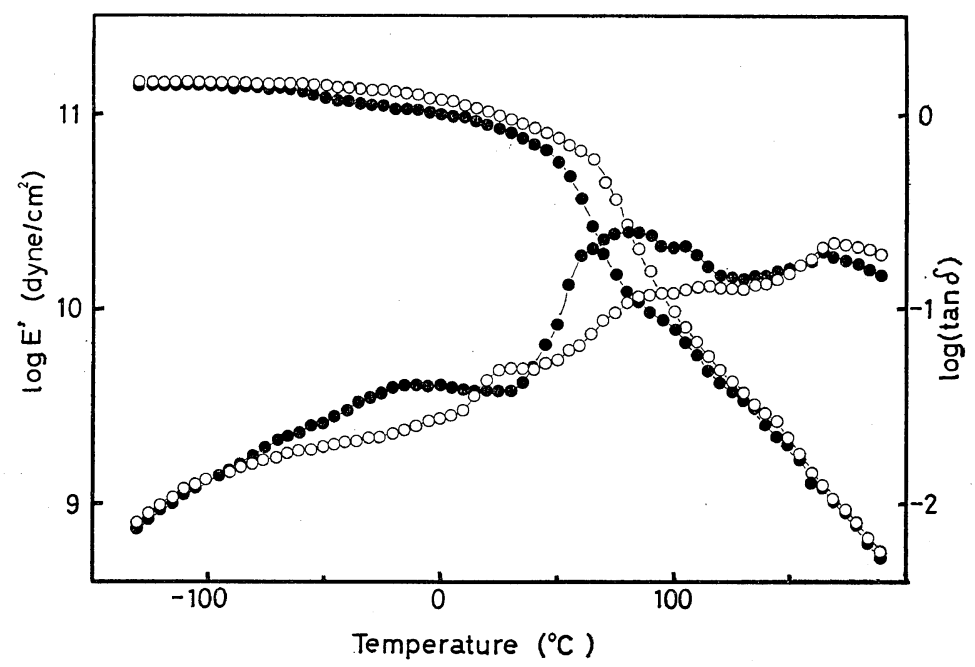

Figure 1. Temperature dependence of dynamic Young's modulus $E^{\prime}$ and $\tan \delta$ for the untreated and heat-treated PVA samples (at $5 \mathrm{~Hz}$ ): e, untreated PVA; $\bigcirc$, heattreated PVA at $120^{\circ} \mathrm{C}, 3 \mathrm{hr}$. 


\section{RESULTS}

The temperature dependences of the dynamic Young's modulus $E^{\prime}$ and $\tan \delta$ measured at $5 \mathrm{~Hz}$ for the untreated and heat-treated PVA samples are shown in Figure 1. It can be seen for untreated PVA that there are four dispersions, located at about $170^{\circ}, 110^{\circ}, 80^{\circ}$, and $-10^{\circ} \mathrm{C}$, in the temperature range investigated. They are called hereafter the $\alpha_{\mathrm{c}^{-}}, \beta_{\mathrm{c}^{-}}, \alpha_{\mathrm{a}^{-}}$, and $\beta_{\mathrm{a}}$-loss peaks respectively in the order of decreasing temperature. ${ }^{8}$ After the heat treatment, the $\alpha_{\mathrm{c}^{-}}$ loss peak at $170^{\circ} \mathrm{C}$ increased in height with a slight shift to higher temperature and the $\beta_{\mathrm{c}}$-loss peak at $110^{\circ} \mathrm{C}$ almost disappeared. Both the $\alpha_{\mathrm{a}}$-loss peak at $80^{\circ} \mathrm{C}$ and the $\beta_{\mathrm{a}}$-loss peak at $-10^{\circ} \mathrm{C}$ decreased in their height and shifted to higher temperatures. These changes in each peak may suggest some rearrangements of molecular organization because of the heat treatment.

Figure 2 shows the $\tan \delta$ curves at $5 \mathrm{~Hz}$ for the PVA and partially acetylated samples. By

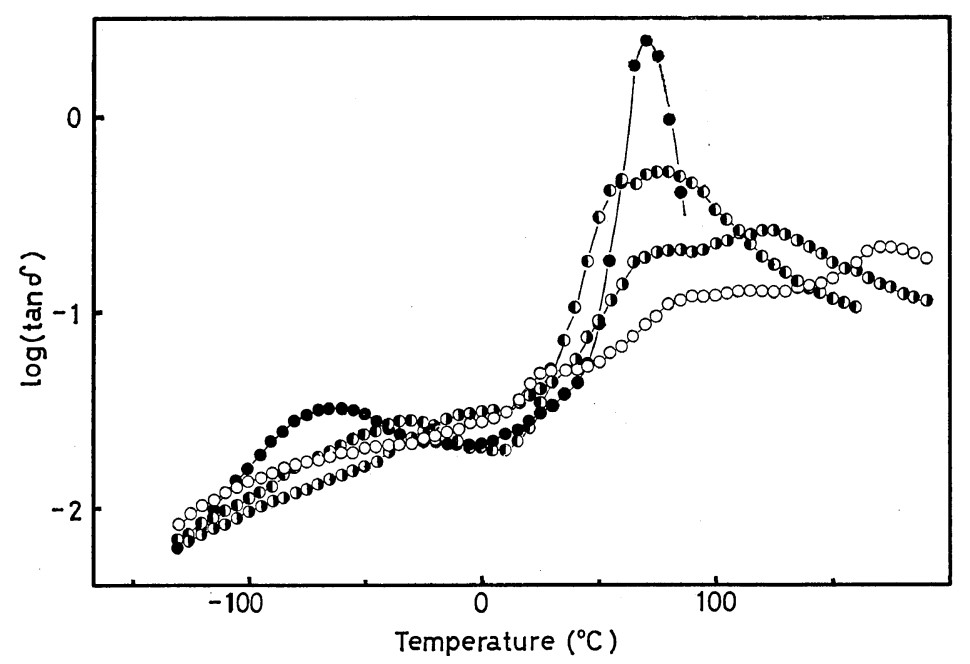

Figure 2. Temperature dependence of $\tan \delta$ for the variously acetylated PVA samples (at $5 \mathrm{~Hz}$ ): О, PVA; PVAc-1; D, PVAc-12; ๑, PVAc-38.

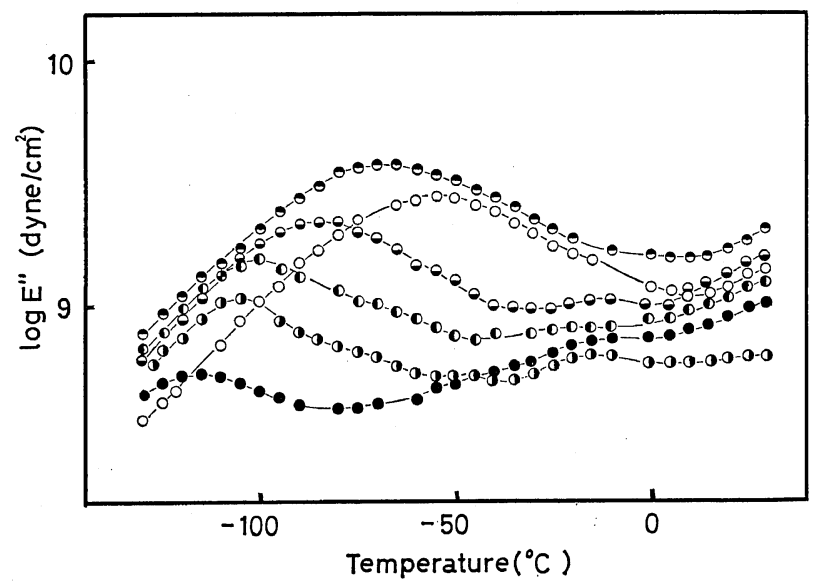

Figure 3. Temperature dependence of loss modulus $E^{\prime \prime}$ in the $\beta_{\mathrm{a}}$-loss peak region for the highly acetylated samples (at $5 \mathrm{~Hz}$ ): $\bigcirc$, PVAc-28; $\Theta$, PVAc-38; $\ominus$, PVAc59; PVAc-69; PVAc-80; ๑, PVAc-100. 
partial acetylation of PVA, each loss peak was shifted to lower temperatures, while their heights increased. The effects on the $\alpha_{\mathrm{c}^{-}}$and $\beta_{\mathrm{c}}$-peak regions are especially remarkable: for the partially acetylated samples, only one peak was observed in this temperature region and it shifted to the low temperature side and increased its height, upon increasing the degree of acetylation. This peak finally coalesced with the $\alpha_{\mathrm{a}}$-loss peak at about $70^{\circ} \mathrm{C}$ in the PVAc-38 sample. But for the more highly acetylated samples, accurate measurements above the $\alpha_{\mathrm{a}}$-peak temperature were impossible owing to the remarkable softening of the samples. Figure 3 shows the temperature dependence of the loss mudulus $E^{\prime \prime}$, at $5 \mathrm{~Hz}$ in the $\beta_{\mathrm{a}}$-peak region of the highly acetylated samples. The peak temperature of the completely acetylated sample agrees well with that corresponding to the motions of the acetyl side groups. ${ }^{17-20}$ The effects of partial acetylation of PVA on each peak temperature are summarized in Figure 4. The introduction of a small number of acetyl groups brings about a noticeable low temperature shift of each loss peak. With further increase of the degree of

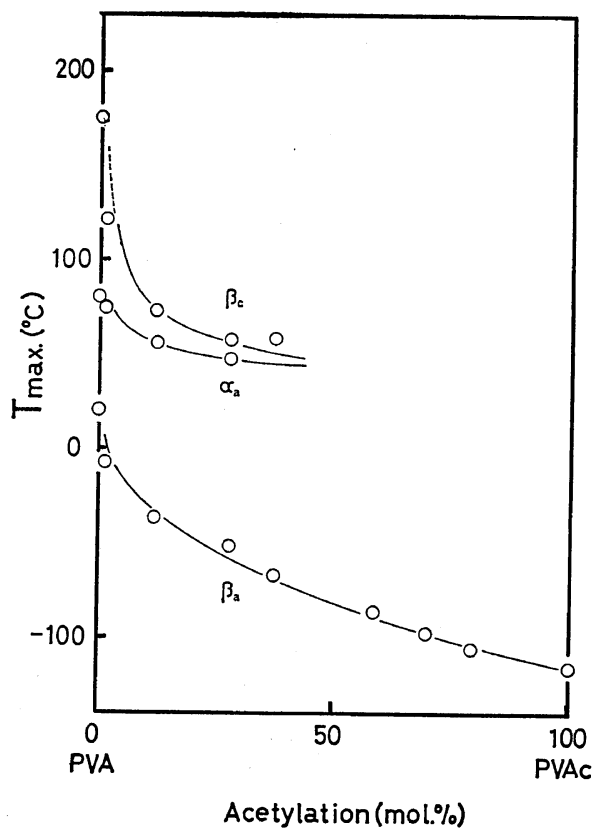

Figure 4. Dependence of the temperatures of each loss peak ( $\left.\tan \delta_{\max }\right)$ on the degree of partial acetylation of PVA (at $5 \mathrm{~Hz}$ ).

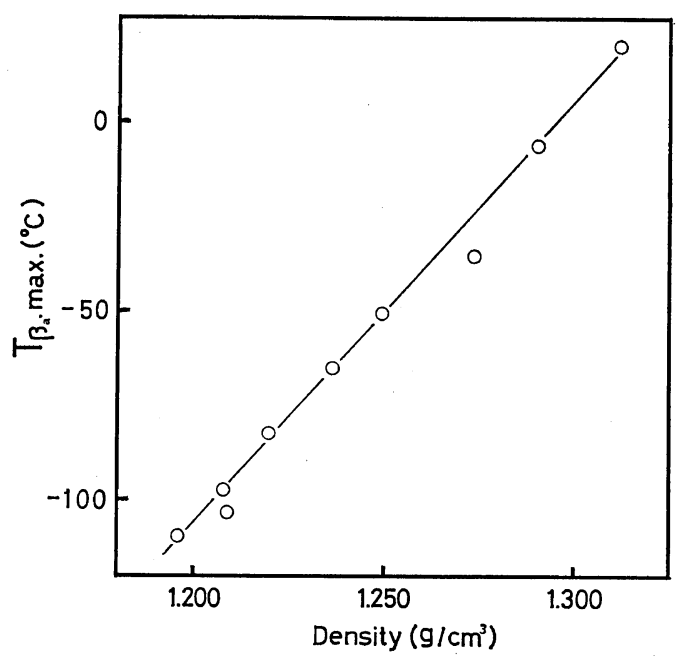

Figure 5. Dependence of the $\beta_{\mathrm{a}}$-peak temperature $\left(\tan \delta_{\max }\right)$ on the density for the variously acetylated samples (at $5 \mathrm{~Hz}$ ).

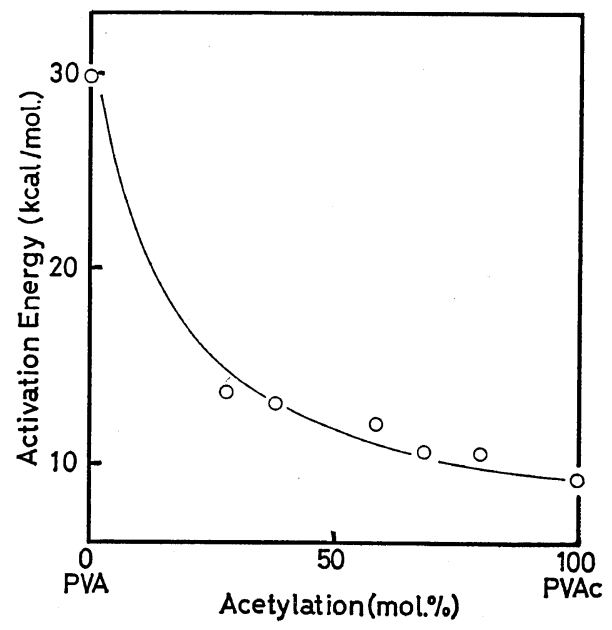

Figure 6. Dependence of the apparent activation energy for the $\beta_{\mathrm{a}}$ process on partial acetylation of PVA.

acetylation, each loss peak tends to split into two peaks, which correspond to the microBrownian motions of the main chain segments and the reorientational motions of the side chain acetyl groups of PVAc, respectively. Further, as is shown in Figure 5, the peak temperature of the $\beta_{\mathrm{a}}$ dispersion was linearly shifted to lower temperatures with decreasing density as the acetylation proceeded. 
Figure 6 shows the dependence of the apparent activation energy for the $\beta_{\mathrm{a}}$ dispersion on the partial acetylation of PVA; the values of $10-15$ $\mathrm{kcal} / \mathrm{mol}$ for the highly acetylated samples are compatible with the values obtained for the reorientational motions of the side chain acetyl groups of PVAc. ${ }^{17-20}$

\section{DISCUSSION}

As is generally known, the thermal treatment of a crystalline polymer such as PVA leads to an increase in its crystallinity and also in the perfectness of its crystalline structure. ${ }^{3}$ It is obvious that the increase in the density upon annealing is consistent with the above generalizations about the thermal treatment. From the results that the height of the $\alpha_{\mathrm{c}}$-loss peak was increased by heat treatment, as shown in Figure 1, and that the apparent activation energy estimated from the frequency dependence had a value as high as about $200 \mathrm{kcal} / \mathrm{mol}$, this $\alpha_{\mathrm{c}}$ dispersion can be ascribed to the motions associated with the crystalline phase. In the present case, however, it is not possible to determine whether this process can be ascribed to the intercrystalline or the intracrystalline process.

On the other hand, the three remarkable changes upon partial acetylation, i.e., the shift to lower temperatures of the $\beta_{\mathrm{c}}$-loss peak accompanying the increase in height, the disappearance of the $\alpha_{\mathrm{c}}$-loss peak, and the decrease in density, can be considered to be interpreted by the following mechanisms. Considering the fact that the introduction of a substituent into the PVA chains generally reduces the orientation of the backbone chains, the bulky acetyl groups introduced randomly and partially into the PVA chains conspicuously lower the possibility of the hydrogen bond formation performing the main role in the crystallization of the PVA chains. ${ }^{1}$ That is, the presence of a small number of acetyl groups may prevent the normal crystallization of the PVA chains. Consequently, in the partially acetylated samples, the formation of an imperfect crystalline phase involving many crsytal defects and vacancies would predominate and also the motions in such a phase would occur more easily. As the degree of acetylation increased, the fraction of such an imperfect crystalline phase would be increased and the polymer structure would be successively changed from semicrystalline to quasicrystalline to completely amorphous. The observed coalescence of the $\beta_{\mathrm{c}^{-}}$and $\alpha_{\mathrm{a}}$-loss peaks in the highly acetylated samples implies that the molecular motions in the imperfect crystalline and amorphous phases became indistinguishable from each other. As for the $\alpha_{\mathrm{c}}$ and $\beta_{\mathrm{c}}$ dispersions, Nakatani and others have reported similar behaviors following changes in the polymer nature by partial formylation of PVA. $^{21}$ To do the full interpretation of these crystalline dispersions, however, it still seems necessary to make more systematic studies taking account of the lamellar structure of PVA. ${ }^{22}$

It is well known that the presence of a diluent and/or bulky pendant side group increases the chain mobility and consequently reduces the glass transition temperature of a polymer., ${ }^{3,21}$ The shift to a lower temperature and the increase in the height of the $\alpha_{\mathrm{a}}$-loss peak in the slightly acetylated samples, as shown in Figures 2 and 4, are nothing but the result of the introduced bulky acetyl groups acting as an internal plasticizer upon the amorphous phase. Also, the results obtained here must involve the effect of lowering the crystallinity due to the bulky acetyl groups introduced, i.e., the acetyl groups may lower the possibility of formation of the hydrogen bonds between the neighboring chains, which strongly restrict the segmental motions of the backbone chains. Consequently, it can be deduced that molecular motions of the chain segments in the amorphous phase become more enhanced, according to the decrease in the numbers of $\mathrm{OH}$ groups and the increase in the separation among the polymer chains, as the degree of acetylation is increased.

The $\beta_{\mathrm{a}}$-loss peak, which is found at about $20^{\circ} \mathrm{C}$ for PVA, has been ascribed to the local relaxational mode of the backbone chain in the frozen state. ${ }^{8}$ More especially, it is thought to originate from the local motions of the chain segments involving the inter- and intramolecular hydrogen-bonded portions. Also, for the moistured PVA, the $\beta_{\mathrm{a}}$-loss peak was shifted to the lower temperature side; this corresponds to the increase in the local chain mobility due to the breakdown of these hydrogen bonds in the water- 
accessible portion with increasing moisture. , $16^{16}$ As shown in Figures 2 and 4, the observed shift to the lower temperature side of the $\beta_{\mathrm{a}}$-loss peak upon partial acetylation may be ascribed to an increase in the local mobility of the backbone chains because of the bulky acetyl groups introduced. That is, the possibility of the formation of the hydrogen bonds which restrict the local motions of the backbone chains may be decreased by partial acetylation, in a way similar to the case of the wet PVA. Furthermore, the shift may also be influenced by the increase in the imperfect crystalline phase caused by the introduced acetyl groups, as mentioned in the discussion of the $\beta_{\mathrm{c}}$ dispersion.

On the other hand, in the highly acetylated samples, the observed $\beta_{a}$-loss peak can be ascribed, in large part, to the reorientational motions of the acetyl side groups, although for pure PVAc the corresponding peak should first be observed at about $-120^{\circ} \mathrm{C}$ (at $5 \mathrm{~Hz}$ ). ${ }^{17-20}$ More precisely, the reorientational motions of the acetyl side groups are strongly restricted and this local restriction on the $\beta_{\mathrm{a}}$ process is greatly decreased upon acetylation. This interpretation is also supported by the remarkable decrease in the activation energy for the $\beta_{\mathrm{a}}$ process shown in Figure 6. Thus, it can be concluded that the acetyl side groups in the partially acetylated PVA cannot rotate independently of the backbone chain, owing to the intense braking effects caused by the hydrogen bonds. Moreover, it is very interesting that there is a linear relation between the $\beta_{2}$-peak temperatures and the sample densities, as shown in Figure 5, notwithstanding the appreciable existence of the crystalline part in the lightly acetylated samples. This suggests that the $\beta_{\mathrm{a}}$ process originates from the local motions of the backbone chains and/or the restricted reorientational motions of the pendant acetyl groups, and that the local free volume for the $\beta_{a}$ process increases with increasing the degree of acetylation. Consequently, the $\beta_{\mathrm{a}}$ dispersion observed in the partially acetylated PVA can be interpreted in terms of a transformation of the local motions of the backbone chain, including the hydrogenbonded portion and/or the imperfect crystalline region, to the restricted reorientational motions of the acetyl side groups, as the degree of acetylation was increased. This transformation is similar to the merging of the $\beta_{\mathrm{c}}$-loss peak into the $\alpha_{\mathrm{a}}$-loss peak in the highly acetylated samples. Therefore, in view of the similarity of their behaviors under partial acetylation, the mechanisms of the $\beta_{\mathrm{c}}$ and $\beta_{\mathrm{a}}$ processes seem to be interrelated. Finally, the small and broad loss peak observed at about $-20^{\circ} \mathrm{C}$ for the highly acetylated samples may be conceivably due to an impurity; this peak is thus designated as $\beta_{\mathrm{w}}$ dispersion, because it is probably due to a small amount of left-over water.

\section{REFERENCES}

1. C. W. Bunn, Nature, 161, 929 (1948).

2. S. Satoh, R. Chūjō, T. Ozeki, and E. Nagai, Rept. Progr. Polym. Phys. Japan, 5, 251 (1962).

3. N. G. McCrum, B. E. Read, and G. Williams, "Anelastic and Dielectric Effects in Polymeric Solids," John Wiley \& Sons Inc., New York, N.Y., 1967, Chapter 9.

4. S. Kurosaki and T. Furuyama, J. Polym. Sci., 43, 137 (1960).

5. Y. Ishida, Y. Tanaka, and M. Takayanagi, Kolloid-Z., 168, 121 (1960).

6. R. C. Laible and H. M. Morgan, J. Polym. Sci., 54, 53 (1961).

7. S. Onogi, K. Sasaguri, T. Adachi, and S. Ogihara, ibid., 58, 1 (1962).

8. M. Takayanagi, Mem. Fac. Eng. Kyusyu Univ., 23, 41 (1963).

9. A. Nagai and M. Takayanagi, Rept. Progr. Polym. Phys. Japan, 7, 249 (1964).

10. T. Kajiyama, S. Togami, Y. Ishida, and $\mathbf{M}$. Takayanagi, J. Polym. Sci., Part B, 3, 103 (1965).

11. A. Nagai and M. Takayanagi, Kogyo Kagaku Zasshi (J. Chem. Soc. Japan, Ind. Chem. Sect.), 68, 836 (1965).

12. R. P. Gupta and R. C. Laible, J. Polym. Sci. Part A, 3, 3951 (1965).

13. K. Fujino, T. Horino, K. Miyamoto, and H. Kawai, J. Colloid Sci., 16, 411 (1961).

14. F. Fujimoto and K. Hirabayashi, Bull. Inst. Chem. Res. Kyoto Univ., 24, 92 (1951).

15. H. Matsuda, S. Ishiguro, K. Naraoka, and A. Kotera, Kobunshi Kagaku (Chem. High Polymers), 12, 10 (1955).

16. H. Ochiai, unpublished results.

17. H. Thurn and K. Wolf, Kolloid-Z., 148, 16 (1956). 
H. Ochiai, H. FujiI, M. Watanabe, and H. Yamamura

18. K. Hikichi and J. Furuichi, Rept. Progr. Polym. Phys. Japan, 4, 69 (1961).

19. Y. Ishida, M. Matsuo, and K. Yamafuji, Kolloid-Z. Z. Polym., 180, 108 (1962).

20. V. Frosini and A. E. Woodward, J. Polym. Sci.
Part A-2, 7, 525 (1969).

21. M. Nakatani, K. Iijima, A. Suganuma, and H. Kawai, J. Macromol. Sci.-Phys., B2, 55 (1968).

22. K. Tsuboi, ibid., B2, 603 (1968). 